\title{
Amantadine Variants Activity Against Multiple Influenza A Viruses
}

Marianna Stampolaki, ${ }^{1}$ Christina Tzitzoglaki, ${ }^{1}$ Christos Liolios, ${ }^{1}$ Anja Hoffmann, ${ }^{2}$ F. Brent Johnson, ${ }^{4}$ loannis Stylianakis, ${ }^{1}$ Roland Zell, ${ }^{2}$ Andreea L. Turcu, ${ }^{3}$ Patrick Schmerer, ${ }^{2}$ Kristin Döring, ${ }^{2}$ Chunlong Ma, ${ }^{5}$ Ivi Theodosia Antoniadou, ${ }^{6}$ David Fedida, ${ }^{7}$ Fransesc Sureda, ${ }^{8}$ Stephen A. Wharton, ${ }^{9}$ Jun Wang, ${ }^{5}$ Santiago Vázquez, ${ }^{3}$ Michaela Schmidtke, ${ }^{2}$ Antonios Kolocouris $1,{ }^{*}$

${ }^{1}$ Laboratory of Medicinal Chemistry, Section of Pharmaceutical Chemistry, Department of Pharmacy, National and Kapodistrian University of Athens, Panepistimiopolis-Zografou, 15771, Athens, Greece

2 Jena University Hospital, Department of Medical Microbiology, Section Experimental Virology, CMB Building, R. 443, Hans Knoell Str. 2, D-07745 Jena, Germany

${ }^{3}$ Laboratori de Química Farmacèutica (Unitat Associada al CSIC), Facultat de Farmàcia i Ciències de I'Alimentació, and Institute of Biomedicine (IBUB), Universitat de Barcelona, Av. Joan XXIII, 27-31, Barcelona, E-08028, Spain

${ }^{4}$ Department of Microbiology and Molecular Biology, Brigham Young University, Provo, UT 84602, United States

${ }^{5}$ Department of Pharmacology and Toxicology, College of Pharmacy, University of Arizona, Tucson, Arizona 85721, United States; Present address: Department of Medicinal Chemistry, Ernest Mario School of Pharmacy Rutgers, The State University of New Jersey, 160 Frelinghuysen Road Piscataway, NJ 08854-8020

${ }^{6}$ Laboratory of Pharmaceutical Technology, Section of Pharmaceutical Technology, Department of Pharmacy, National and Kapodistrian University of Athens, Panepistimiopolis-Zografou, 15771, Athens, Greece; Present address: Biomedical Research Foundation of the Academy of Athens, 4 Soranou Ephessiou Str., 11527 Athens, Greece

7 Department of Anesthesiology, Pharmacology and Therapeutics, University of British Columbia, Vancouver, British Columbia, V6T 1Z3, Canada

${ }^{8}$ Department of Biochemistry and Biotechnology, Faculty of Medicine and Life Science, University Rovira i Virgili, Reus, Spain 
${ }^{9}$ Crick Worldwide Influenza Centre, The Francis Crick Institute, 1 Midland Road, London NW1 1AT, UK

Keywords: amantadine, antiviral activity, cocktail, electrophysiology, endosome neutralizers, haemagglutinin, influenza $A$, ion channel, M2 WT protein, M2 L26F protein, M2 S31N protein, M2 V27A protein, neurotoxicity protein, point mutations, synthesis 


\section{Abstract}

Future pandemic influenza necessitates the development of new drugs against the current circulating, amantadine and rimantadine drugs resistant, influenza A M2 S31N viruses. The possibility of an antigenic shift to M2 S31 necessitates ranking the biological activities of amantadine variants. Several amantadine variants have been tested by different laboratories, but various M2 wild type influenza A strains have been used with different sensitivity against amantadine and the unambiguous comparison between potencies is not straightforward. Here, we compared the anti-influenza activities of 57 synthetic amantadine variants against influenza $A$ WSN/33 viruses with amantadine-sensitive M2 WT, with a range of over three digits providing a reference set of potencies for structure-activity relationships, and amantadine-resistant M2 S31N proteins (and observed no potent compounds). 17 compounds were selected and tested against M2 L26F, V27A, A30T, G34E viruses. We tested few reference compounds using electrophysiology and explored point mutations which both showed that $\mathrm{M} 2$ is the target of potent antiviral potency against the M2 WT, L26F, V27A viruses. Major findings are: (a) Several amantadine variants from Kolocouris group block only M2 WT and M2 L26F-mediated proton current and the corresponding viruses replication. (b) A compound from Vazquez's group is a triple blocker of M2 WT, L26F, V27A channels and viruses replication. (c) A compound from Vazquez's group blocks only M2 L26 channel and virus replication. (d) Several compounds from Kolocouris group have potent activity against several influenza A M2 WT and three M2 S31N viruses, eg. the pandemic A/H1N1/California/07/2009 (H1N1pdm09) or A/H1N1/PuertoRico/08/1934 without blocking M2 S31N. The compounds and their cocktails while not to be more toxic than amantadine might be useful for re-purposing of amantadine class of drugs in the case (i) of the prevalence of M2 L26F and or M2 V27A strains (ii) of an antigenic shift of the virus to M2 WT and (iii) because they inhibited a broad panel of M2 WT and M2 S31N viruses including the H1N1pdm09). (d) We showed that the mechanism of antiviral activity against A/California/07/2009 or A/PR/08/1934 and possibly also M2 WT viruses compared to WSN/33 viruses is not due to inhibition of an early stage of virus infection or a late stage of $M 2$ channel function during endocytosis or inhibition of $\mathrm{HA}$ binding to host cells or a different $\mathrm{pH}$ for $\mathrm{HA}$ fusion or a lysosomotropic effect. 


\section{Introduction}

Amantadine $(A m t, 1)$ and rimantadine $(\operatorname{Rim}, 2)($ Table 1$)$ are blockers of proton transport by $M 2$ ion channel of influenza A wild type (WT) virus, 1,2,4 and were clinically approved as prophylactics and therapeutics against influenza A viruses. ${ }^{3}$ Several groups published high resolution structures and data from mutation experiments showing that M2 protein channel is blocked by $\mathbf{1}$ and $\mathbf{2}$ via a M2 pore-binding mechanism. ${ }^{4-9}$ Additionally, amantadine variants, especially those with hydrophobic adducts, can act as lysosomotropic drugs, ${ }^{10}$ which accumulate in intracellular vesicles through membrane permeation by the electroneutral form and increase intravesicular $\mathrm{pH}$, causing endosome and/or trans-Golgi network neutralization and inhibition of viral reproduction. ${ }^{11}$ Noteworthy, SARS-CoV-2 is inhibited by chloroquine, probably because it acts as a lysosomotropic drug. 12

Resistance to M2 WT proton channel drugs is associated with mutations in the TM domain of the M2 protein. ${ }^{13-15}$ The homotetrameric structure of the $M 2$ channel places constraints on the types of drug-resistant mutations that can be accommodated. ${ }^{16,17}$ The amino acid substitutions L26F, V27A, A30T, G34E and S31N were shown to confer cross-resistance to Amt (1) and Rim (2) against influenza A viruses. ${ }^{18-20}$ The vast majority, $95 \%$, of resistant viruses bear the S31N substitution in $\mathrm{M} 2,1 \%$ have V27A, and L26F, A30T, and G34E are rare. ${ }^{21,22}$ The substitution V27A most often emerged under drug selection pressure. ${ }^{23,18}$ The other mutations confer Amt (1) resistance but this is not a result of the $A m t(1)$ drug selection pressure. ${ }^{23}$ The $M 2$ S31N mutant is a natural mutation and one of the most conserved in viral proteins among currently circulating influenza $A$ viruses that happens to maintain nearly identical channel function as the M2 WT but is resistant to Amt (1). The presence of L26F, V27A, and particularly S31N in influenza A viruses circulating worldwide pushes the search for novel ion channel blockers with stronger, preferably resistanceovercoming activity.

After early work on potent synthetic amantadine analogues, ${ }^{24}$ many other amantadine variants, 25-30 polycyclic cage amines and their guanidino analogues have been synthesized, ${ }^{31,32} \mathrm{eg}$. 
compounds 3-8. Compounds 3 and 8 block the M2 WT- proton mediated current. Compounds 4, 6 31 block both M2 WT- and M2 V27A-proton mediated current and inhibit these viruses replication. Compounds $5^{31}$ and $7^{33}$ are blockers of the M2 WT, V27A, L26F channels. Although these compounds do not block M2 S31N channel and new derivatives that block M2 S31N have been developed, ${ }^{34,35}$ they are still valuable due to the unpredictable mutations of the virus M2 S31 channel in current epidemics.

Table 1. Chemical structures of representative amantadine variants and analogues and their potencies against M2 WT and the amantadine resistant M2 V27A, M2 L26F and M2 S31N mutant channels.

\begin{tabular}{|l|c|c|c|c|}
\hline & \multicolumn{3}{|c|}{ Compound No/Structure } \\
\hline & 1 & 2 & \\
\hline & & & \\
\hline
\end{tabular}

n.a., not active 
While several amantadine variants have been tested by different laboratories, various strains of M2 WT virus have been used with different sensitivity against $A m t$ (1). 24,25,30,31 Subsequently, the unambiguous comparison between potencies is not straightforward. We provided here a data set of 57 amantadine variants with in vitro antiviral potencies (Figure 1) against the WSN/33-M2-N31S virus strain, which has as natural strain the $A m t$ (1)-resistant influenza virus A/WSN/33 (M2 N31). In the A/WSN/33 M2-N31S strain the M2 key amino acid residues 26, 27, 30, 31 and 34 of A/WSN/33 M2-S31 are identical with the Udorn strain with M2 WT protein. From now on we will use the term M2 WT for the M2 N31S channel of the A/WSN/33 M2-N31S strain. We selected and tested 17 representative amantadine variants against the Amt (1)-resistant influenza viruses with M2 L26F, M2 V27A, M2 A30T, M2 G34E, i.e. the A/WSN/33 M2-N31S L26F, A/WSN/33 M2-N31S V27A, A/WSN/33 M2-N31S A30T, A/WSN/33 M2-N31S G34E viruses. ${ }^{17}$ To render the in vitro inhibitory activities against influenza A comparable we confirmed that the compounds act at the same protein target. Thus, for a subset of compounds we carried out electrophysiology (EP) experiments and showed that only the active compounds block the $\mathrm{M} 2$ channel-mediated proton current. Since these compounds may be useful as future antivirals, we also studied the mutations that few representative compounds caused to M2TM WT pore under conditions of drug pressure. 


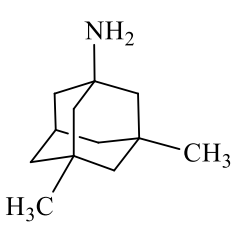

9

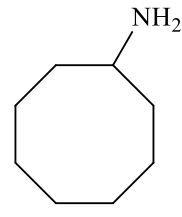

10<smiles>[R]C([R3])(N)N</smiles>

11: $\mathrm{R}_{1}=\mathrm{Et}, \mathrm{R}_{2}=\mathrm{Pr}, \mathrm{R}_{3}=\mathrm{Bu}$

12: $R_{1}=\operatorname{Pr}, R_{2}=\operatorname{Pr}, R_{3}=P r$

13: $\mathrm{R}_{1}=\operatorname{Pr}, \mathrm{R}_{2}=\operatorname{Pr}, \mathrm{R}_{3}=\mathrm{Bu}$

14: $R_{1}=\operatorname{Pr}, R_{2}=P r, R_{3}=i B u$

15: $R_{1}=\operatorname{Pr}, R_{2}=\operatorname{Pr}, R_{3}=t B u$
16: $\mathrm{R}_{1}=\mathrm{Pr}, \mathrm{R}_{2}=\mathrm{Bu}, \mathrm{R}_{3}=\mathrm{Bu}$

17: $R_{1}=B u, R_{2}=B u, R_{2}=B u$

18: $R_{1}=B u, R_{2}=B B u, R_{3}=i B u$

19: $R_{1}=i B u, R_{2}=i B u, R_{3}=i B u$

20: $R_{1}=M e, R_{2}=M e, R_{3}=$ neopentyl

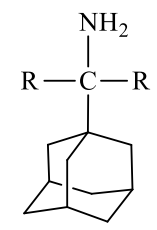

21: $\mathrm{R}=\mathrm{Me}$

22: $\mathrm{R}=\mathrm{Et}$

23: $\mathrm{R}=\mathrm{n}-\mathrm{Pr}$

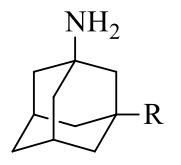

30: $\mathrm{R}=i$-Pr

31: $\mathrm{R}=\mathrm{F}$

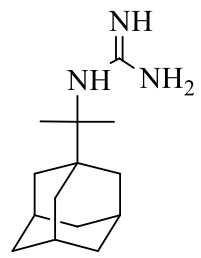

24

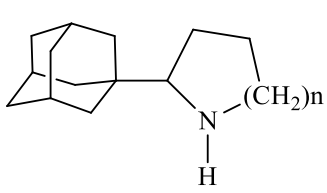

25: $\mathrm{n}=1$

26: $n=2$

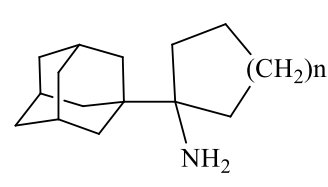

27: $\mathrm{n}=1$

28: $n=2$

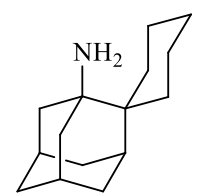

29

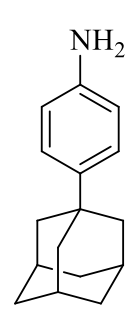

32

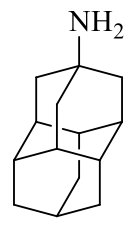

33

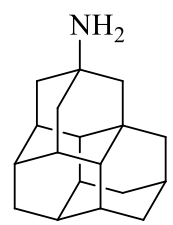

34<smiles>[R]C1(N)C2CC3CC(C2)CC1C3</smiles>

35: $\mathrm{R}=\mathrm{H}$

36: $\mathrm{R}=\mathrm{Me}$

37: $\mathrm{R}=\mathrm{Et}$

38: $\mathrm{R}=\mathrm{n}-\mathrm{Pr}$

39: $\mathrm{R}=\mathrm{n}-\mathrm{Bu}$

40: $\mathrm{R}=\mathrm{i}-\mathrm{Bu}$

41: $\mathrm{R}=\mathrm{n}-\mathrm{Hex}$

42: $\mathrm{R}=\mathrm{Ph}$

43: $\mathrm{R}=\mathrm{CH}_{2} \mathrm{Ph}$

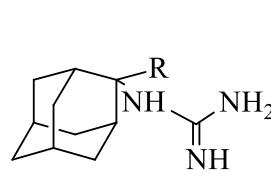

44: $\mathrm{R}=\mathrm{Me}$ 45: $\mathrm{R}=n-\mathrm{Pr}$

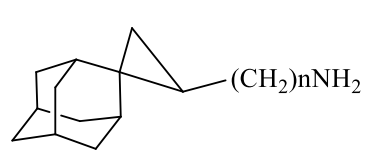
46: $n=0$
47: $n=1$

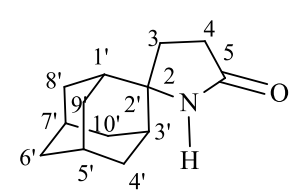

48

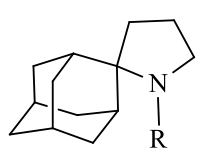

49: $\mathrm{R}=\mathrm{H}$

50: $\mathrm{R}=\mathrm{Me}$

51: $\mathrm{R}=\mathrm{CH}_{2} \mathrm{CH}_{2} \mathrm{NH}_{2}$

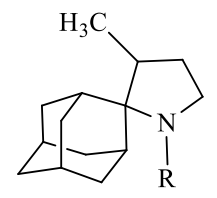

52: $\mathrm{R}=\mathrm{H}$

53: $\mathrm{R}=\mathrm{Me}$

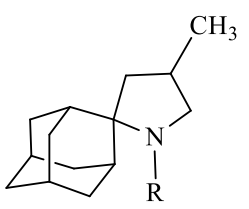

54: $\mathrm{R}=\mathrm{H}$ 55: $\mathrm{R}=\mathrm{Me}$

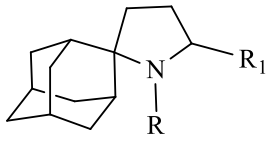

56: $\mathrm{R}=\mathrm{H}, \mathrm{R} 1=\mathrm{Me}$ 57: $\mathrm{R}=\mathrm{Me}, \mathrm{R} 1=\mathrm{Me}$ 58: $\mathrm{R}=\mathrm{H}, \mathrm{R} 1=\mathrm{Et}$

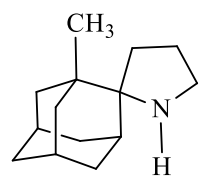

59

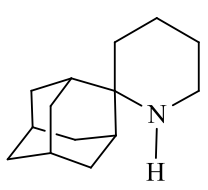

60

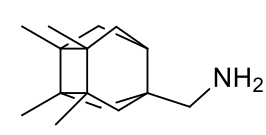

61

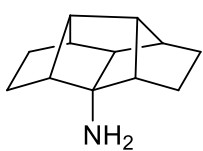

62

Figure 1. Chemical structures of the 57 amantadine variants tested against influenza A M2 WT and M2 S31N (WSN/33 N31S and WSN/33, respectively).

Amt (1) or $\operatorname{Rim}(2)$ are inactive against influenza A/H1N1/Calif/07/2009 (A/Calif/07/09 or H1N1pdm09), A/H1N1/PuertoRico/8/1934 (A/PR/8/34 or APR8), A/H1N1/WS/33 having M2 channel with asparagine at position-31 of $M 2$ and active against $A / H 3 N 2 / V i c t o r i a / 3 / 75$ and A2/H2N2/Taiwan/1/64 having M2 channel with serine-31 but also against the A/H1N1/PR/8/1934 
strain which has the M2 A30T S31N protein. Strikingly, few amantadine variants inhibited the A/H1N1/PR/8/1934 (A30T/S31N) ${ }^{37,38}$ and the pandemic strain A/California/07/2009 (S31N) 28 influenza A strains, without blocking efficiently the M2 S31N channel according to isothermal titration calorimetry, EP, solid state NMR chemical shifts, and molecular dynamics simulations, ${ }^{39,40}$ which also showed that M2 N31 M2 mutation abolishes the lipophilic pocket enclosed by V27 side chains causing a propensity for Amt (1) variants to change orientation pointing ammonium group $\mathrm{N}$-ward in the M2 S31N pore compared to their tight C-ward orientation in the M2 WT pore. ${ }^{8,9}$

To explore this biological activity for a larger set of amantadine variants, we tested many of the compounds against the $\mathrm{A} / \mathrm{H} 1 \mathrm{~N} 1 /$ California/07/2009, the $\mathrm{A} / \mathrm{H} 1 \mathrm{~N} 1 /$ PuertoRico/8/1934, and the A/H1N1/WS/33 viruses, all having M2 S31N proteins. Compounds were also tested against the influenza A M2 WT viruses A/H3N2/Victoria/3/75 (A/Victoria/3/75) and A2/H2N2/Taiwan/1/64.

\section{Results and Discussion}

\section{Compounds set}

Memantine (9) and cyclooctanamine (10), are tert-alkyl amines analogues of amantadine 11-20. Compounds 21-28 include a linear alkyl or heterocyclic or carbocyclic substitution and are $\operatorname{Rim}(\mathbf{2})$ analogues. Compounds $\mathbf{2 9}$, and $\mathbf{3 0}$ or $\mathbf{3 1}$ have a spirocyclohexyl at C-2 position and a F or iPr group at C-3 position of adamantane, respectively. Compounds 32, and 33, $\mathbf{3 4}$ include a phenyl as linker between amino group and 1-adamantyl group, and diamantyl, triamantyl, respectively. Compounds 35-60 have an amino group at C-2 position and also include additional carbon atoms in the form of a linear alkyl or saturated 3-, 5-, 6-membered heterocycle. Compounds 35-45 include an alkyl at C-2 adamantane carbon, 46, 47 contain a spirocyclopropyl, 48-59 contain a spiropyrrolidine while 60 a spiropiperidine ring. Cage amines 61, 62 were tested as polycyclic analogues. Guanidine derivatives 24, 44, 45 were also included after the observation that 4 and 8 are potent anti-influenza A compounds. We re-synthesized many of the amantadine variants 
previously reported 8, 21-23, 25-29, 32-43, 46-50, 52-57, 59-62 (see Supporting Information). Several new compounds were synthesized and tested, ie. 11-20, 30, 31, 44, 45, 52-55, 51, 56, 58 (Schemes S1-S6).

\section{Biological activity}

\section{Antiviral activity against WSN/33 and WSN/33-M2-revertant viruses}

Compounds 1, 2-(R), 2-(S), 2-(rac), 9-62 were tested in CPE inhibitory assays ${ }^{41}$ in MDCK cells against two influenza virus strains, having M2 WT and M2 N31 viruses (A/WSN/33 M2 N31S and A/WSN/33 respectively); A/WSN/33 M2 N31S was produced by reverse genetics from A/WSN/33 strain. The $\mathrm{IC}_{50}$ values are shown in Table 2. None of the tested compounds showed potency against the M2 N31 (A/WSN/33) strain. Most of the compounds were potent against M2 WT virus, with 21, 24, 33 endowed with nanomolar inhibitory potency, i.e. IC 50 values $0.03,0.04,0.07 \mu \mathrm{M}$, respectively, very similar to that of $\operatorname{Rim}(2)\left(\mathrm{IC}_{50}=0.02-0.04 \mu \mathrm{M}\right)$ and 9-fold more potent than $A m t$ (1) $\left(I_{50}=0.38 \mu \mathrm{M}\right)$. Of note, the presence of the amino group is crucial for the activity since lactam 28 have Ic50 value $100 \mu \mathrm{M}$. Compounds 25, 36-38, 45-47, 49 and 60 have IC 50 values between 0.1-0.3 $\mu \mathrm{M}$. Compounds $\mathbf{2 2}$, 23, 26, 34, 35, 44, 50, 52, 54-56 have activities in the range of $\mathrm{IC}_{50}$ values 0.5-1 $\mu \mathrm{M}$ and compounds 57, 32, 52 have $\mathrm{IC}_{50}$ values 1-3 $\mu \mathrm{M}$. Compounds 10, 20, 27, 51, 58, 59 have $I C_{50}$ values 4-7 $\mu \mathrm{M}, 30,31$ have $I C_{50}$ values 14.7, $16.2 \mu \mathrm{M}$, and 40, 43 have IC values 22.6, $31.3 \mu \mathrm{M}$, respectively. Compounds 41, 42 are not active. Compared to Amt-(1), the more elongated molecules $\mathbf{2 1}$ and $\mathbf{3 3}$ have the highest potency, similar to $\operatorname{Rim}(\mathbf{2}){ }^{39}$ The enantiomers of $\operatorname{Rim}(\mathbf{2})$ have equal potency to $2-(r a c)$. The effect of moving the amino group from 1- at 2-position does not affect potency (compared 1 with 35) but results in higher toxicity in cells (compared 1 with 35, 36). The installation of an alkyl group from Me to $\mathrm{Pr}$ at the 2-position of 35 does not reduce potency (compare 1 or 35 vs 36-38, Table 2) However, further increase of the alkyl size is detrimental for potency (see compounds 39-43). In particular, compared to 36 longer alkyls (n-butyl or n-hexyl in compounds 39 or $\mathbf{4 1}$, respectively) or branching (i-butyl in compound 40) reduce potency, eg. from 10 -fold and 100 -fold for $n$-Bu and $i$-Bu, respectively, while potency 
disappears in n-hexyl derivative 41. While 2-phenyl substitution, as in 42, leads to an inactive compound, the benzyl substituent, as in $\mathbf{4 3}$, leads to a 46 -fold less potent compound, compared to 1 and 35. The spirocyclopropanamines 46, 47, and the spiropirrolidine 49 and the spiropiperidine 60 have potencies similar to that of $A m t(1)$, in qualitative agreement with previous results with different influenza A strains. ${ }^{25,36}$ The effect of the $\mathrm{N}$-methylation of pyrrolidine ring in compounds $\mathbf{5 0}, \mathbf{5 3}, \mathbf{5 5}, 57$ is the 4 -fold reduction in potency compared to $49,52,54,56$, respectively. The

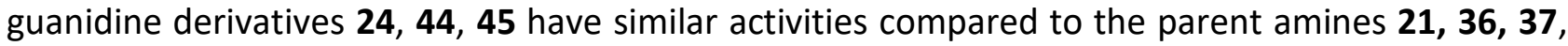
in agreement with previous observations. ${ }^{38}$

It is striking that small changes in structure resulted in remarkable changes in potencies or large changes that did not affect the potency. Such cases are presented when the potency is reduced from ca. $4 \mu \mathrm{M}$ for the $n$-Bu adduct in 39 to $41 \mu \mathrm{M}$ for the $i$-Bu adduct in 40 ; the 30-fold reduction in potency from $0.03 \mu \mathrm{M}$ in $\mathbf{2 1}$ to ca. $1 \mu \mathrm{M}$ to $\mathbf{2 2 , 2 3}$; the 120-fold reduction in potency or potency abolishment in rimantadine analogues $\mathbf{2 7}$ or $\mathbf{2 8}$. However, pyrrolidine analogue of rimantadine $\mathbf{2 5}$ and piperidine analogue 26 have $\mathrm{IC}_{50}$ values 0.19 and $0.90 \mu \mathrm{M}$, respectively. The most serious effect of C-alkylation to potency was observed in pyrrolidine $\mathbf{5 9}$ which reduces potency 10-fold compared to 49 and to memantine (9) which is inactive. The second amino group in spiropyrrolidine $\mathbf{5 1}$ reduces the potency by 10-fold compared to compound $\mathbf{5 1}$. Noteworthy, compared to Amt (1) $\left(\mathrm{IC}_{50}=0.33 \mu \mathrm{M}\right)$ the increased in girth of the cage alkyl in diamantane 34 results only in 2-fold potency reduction $\left(\mathrm{IC}_{50}=0.72 \mu \mathrm{M}\right)$ while the 3-isopropyl-1-amantadine $\mathbf{3 1}$ is mediocre in potency $\left(\mathrm{IC}_{50}\right.$ c.a. $\left.17 \mu \mathrm{M}\right)$. The effect of a single bridgehead fluorination to $A m t(\mathbf{1})$ is similar and 30 has a potency ca. $14 \mu \mathrm{M}$. The acyclic analogues of amantadine 11-19 were inactive except neo-pentylamine (20) with a potency c.a. $5 \mu \mathrm{M}$ in agreement with previous results from EP studies. ${ }^{30}$ Cyclooctylamine has a potency c.a. $7 \mu \mathrm{M}$. 
Table 2. Antiviral activity and cell cytotoxicity against influenza M2 WT ${ }^{a, b}$ virus.

\begin{tabular}{|c|c|c|}
\hline Compound & $I C_{50}(\mu M)^{c}$ & $\mathrm{CC}_{50}(\mu \mathrm{M})^{\mathrm{c}}$ \\
\hline 1 & $0.38 \pm 0.17$ & $>100$ \\
\hline $2-(r a c)$ & $0.04 \pm 0.01^{d, f, 42}$ & $>100$ \\
\hline $2-(R)$ & $0.04 \pm 0.01$ & $>100$ \\
\hline 2-(S) & $0.02 \pm 0.01$ & $>100$ \\
\hline 9 & not active & $101.92 \pm 1.64$ \\
\hline 10 & $6.85 \pm 3.76$ & $>100$ \\
\hline $11-19$ & not active & $>100$ \\
\hline 20 & $5.36 \pm 2.01$ & $>100$ \\
\hline 21 & $0.03 \pm 0.02^{f, 39}$ & $>100$ \\
\hline 22 & $1.01 \pm 0.13^{f, 39}$ & $>100$ \\
\hline 23 & $1.06 \pm 0.23^{f, 39}$ & $71.28 \pm 11.46$ \\
\hline 24 & $0.04 \pm 0.01$ & $>100$ \\
\hline 25 & $0.19 \pm 0.09$ & $>100$ \\
\hline 26 & $0.80 \pm 0.37$ & $>100$ \\
\hline 27 & $4.34 \pm 2.94^{f, 43}$ & $95.16 \pm 4.19$ \\
\hline 28 & not active ${ }^{f, 43}$ & $>100$ \\
\hline 29 & not active & 61.64 \\
\hline 30 & $14.12 \pm 3.72$ & $>100$ \\
\hline 31 & $16.19 \pm 5.36$ & $>100$ \\
\hline 32 & $2.51 \pm 1.24$ & $>100$ \\
\hline 33 & $0.07 \pm 0.02$ & $>100$ \\
\hline 34 & $0.72 \pm 0.33$ & $>100$ \\
\hline 35 & $0.50 \pm 0.38$ & $70.50 \pm 25.31$ \\
\hline 36 & $0.33 \pm 0.10^{f, 43}$ & $25.63 \pm 7.65$ \\
\hline 37 & $0.29 \pm 0.23^{f, 43}$ & $>100$ \\
\hline 38 & $0.34 \pm 0.10^{f, 43}$ & $>100$ \\
\hline 39 & $3.83 \pm 1.71$ & $>100$ \\
\hline 40 & $31.33 \pm 11.88^{d}$ & $>100$ \\
\hline 41 & $>31.6$ & $31.64 \pm 23.27$ \\
\hline 42 & $>100$ & $>100$ \\
\hline 43 & $22.59 \pm 8.58$ & $>100$ \\
\hline 44 & $0.69 \pm 0.26$ & $>100$ \\
\hline 45 & $0.29 \pm 0.14$ & $>100$ \\
\hline 46 & $0.16 \pm 0.09$ & $>100$ \\
\hline 47 & $0.12 \pm 0.06$ & $>100$ \\
\hline 48 & $\sim 100$ & $>100$ \\
\hline 49 & $0.34 \pm 0.08^{f, 43}$ & $>100$ \\
\hline 50 & $0.90 \pm 0.29^{f, 43}$ & $>100$ \\
\hline 51 & $4.33 \pm 2.26$ & $>100$ \\
\hline 52 & $0.68 \pm 0.32$ & $>100$ \\
\hline 53 & $2.98 \pm 1.46$ & $>100$ \\
\hline
\end{tabular}




\begin{tabular}{ccc}
$\mathbf{5 4}$ & $0.85 \pm 0.41$ & $>100$ \\
$\mathbf{5 5}$ & $0.68 \pm 0.32$ & $>100$ \\
$\mathbf{5 6}$ & $0.96 \pm 0.64$ & $>100$ \\
$\mathbf{5 7}$ & $1.92 \pm 1.07$ & $>100$ \\
$\mathbf{5 8}$ & $7.01 \pm 1.84$ & $>100$ \\
$\mathbf{5 9}$ & $6.12 \pm 2.59$ & $>100$ \\
$\mathbf{6 0}$ & $0.34 \pm 0.19$ & $>100$ \\
$\mathbf{6 1}$ & 1.75 & $>100$ \\
$\mathbf{6 2}$ & not active & $>100$ \\
\hline
\end{tabular}

${ }^{a}$ Influenza M2 WT correspond to WSN/33 M2 N31S virus; ${ }^{b}$ all compounds were inactive against M2 N31 virus, i.e. the WSN/33 strain. ${ }^{c}$ mean and standard deviations of the $50 \%$ inhibitory concentration $\left(\mathrm{IC}_{50}\right)$ and the $50 \%$ cytotoxic concentration $\left(\mathrm{CC}_{50}\right)$ of at least three independent assays determined in Madin-Darby canine kidney cells; ${ }^{d}$ compound 40 was considered as less potent inside the series; ${ }^{e}$ n.t., not tested, n.a., not active; the lowest $\mathrm{IC}_{50}$ value is grey shaded and written with bold text and the highest measured $\mathrm{IC}_{50}$ value is grey shaded and written with plain text; ${ }^{f}$ data published in indicated reference.

We selected a few compounds with representative antiviral activity against the M2 WT virus, for testing their potency against other Amt (1) resistant viruses, ie., those carrying the M2 L26F, V27A, A30T and G34E (Table 3). This compounds set include the mid-nanomolar aminoadamantane

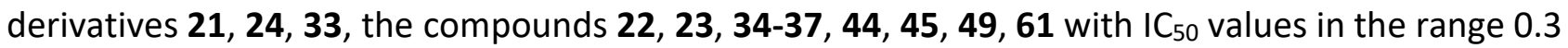
$\mu \mathrm{M}$ to $2 \mu \mathrm{M}$, the compounds 20 and 32 with $\mathrm{IC}_{50}$ values 5.36 and $2.51 \mu \mathrm{M}$, respectively, the compounds 30 and 31 with IC 50 values of c.a. 14.12 and $16.19 \mu \mathrm{M}$, respectively, and the not active, acyclic, primary tert-alkyl amine 18 as well as 29, 62. We observed that compounds $\mathbf{2 1 ,} 24$ and 33 also have low micromolar potencies against the L26F strain and, additionally, diamantane 33 also inhibits V27A, with $I_{50}$ of $15.5 \mu \mathrm{M}$. Compounds 23, 37 and 45 also showed low micromolar potency against the L26F mutant, in the range 0.5-2 $\mu \mathrm{M}$ and 22 and 49 have $\mathrm{IC}_{50}$ values 5.95 and $8.75 \mu \mathrm{M}$, respectively. Neopentylamine $\mathbf{1 8}$, and the aminoadamantane derivatives $\mathbf{2 0}, \mathbf{3 0 - 3 2}$ and 34 are generally not active or have weak potency and 35, 36 and 44 have $\mathrm{IC}_{50}$ values in the range of 16-23 $\mu \mathrm{M}$ ). Except of the weak activity of compound 32 against M2 A30T virus none of the compounds is active against the resistant to Amt (1) strains, M2 A30T and G34E. 
Table 3. Antiviral activity against influenza A M2 L26F, ${ }^{a} \mathrm{~V} 27 \mathrm{~A},{ }^{b} \mathrm{~A} 30 \mathrm{~T},{ }^{c} \mathrm{G} 34 \mathrm{E}{ }^{d}$ viruses. ${ }^{a}$

\begin{tabular}{|c|c|c|c|c|}
\hline \multirow[t]{2}{*}{ Compound } & \multicolumn{4}{|c|}{$I C_{50}(\mu \mathrm{M})^{e}$} \\
\hline & M2 L26F & M2 V27A & M2 A30T & M2 G34E \\
\hline 18 & n.a ${ }^{f}$ & n.a & n.a & n.a \\
\hline 20 & n.a & n.a & n.a & n.a \\
\hline 21 & $0.79 \pm 0.37$ & n.a & n.a & n.a \\
\hline 22 & $5.95 \pm 3.35$ & n.a & n.a & n.a \\
\hline 23 & $0.55 \pm 0.23$ & n.a & n.a & n.a \\
\hline 24 & $1.01 \pm 0.50$ & n.a & n.a & n.a \\
\hline 29 & n.a & n.a & n.a & n.a \\
\hline 30 & $69.45 \pm 28.73$ & n.a. & n.a & n.a. \\
\hline 31 & n.a & n.a & n.a & n.a \\
\hline 32 & $45.30 \pm 10.76$ & n.a. & $40.64 \pm 18.78$ & n.a \\
\hline 33 & $0.74 \pm 0.26$ & $15.46 \pm 6.97$ & n.a & n.a \\
\hline 34 & n.a. & n.a. & n.a. & n.a. \\
\hline 35 & $20.97 \pm 5.00$ & n.a & n.a & n.a \\
\hline 36 & $16.10 \pm 8.72$ & n.a & n.a & n.a \\
\hline 37 & $1.99 \pm 0.74$ & n.a & n.a & n.a \\
\hline 44 & $23.13 \pm 6.34$ & n.a & n.a & n.a \\
\hline 45 & $1.79 \pm 1.05$ & n.a & n.a & n.a \\
\hline 49 & $8.75 \pm 4.72$ & n.a & n.a & n.a \\
\hline 61 & $3.84 \pm 1.99$ & n.a & n.a & n.a \\
\hline 62 & n.a & n.a & n.a & n.a \\
\hline
\end{tabular}

${ }^{a}$ Influenza M2 L26F correspond to A/WSN/33 M2 N31S L26F; ${ }^{b}$ influenza A M2 V27A correspond to A/WSN/33 M2 N31S V27A; ' influenza A A30T correspond to A/WSN/33 M2 N31S A30T; ${ }^{d}$ influenza A M2 G34E correspond to A/WSN/33 M2 N31S G34E; ${ }^{e}$ mean and standard deviations of the $50 \%$ inhibitory concentration (IC $\mathrm{C}_{50}$ ) of at least three independent assays determined in Madin-Darby canine kidney cells; ${ }^{f}$ n.a., not active.

Functional inhibition of $\mathrm{M} 2$ channels by selected amantadine variants

We verified that $M 2$ channel is the protein target for inhibition of influenza $A$ viruses by measuring the blocking effect of few compounds against full length $M 2$ using EP (Table 4, see also Supporting Information ${ }^{36}$ ). We measured the blocking effect against full length M2 protein with a two-electrode voltage clamp (TEVC) assay at $2 \mathrm{~min}$. We tested compounds $\mathbf{2 1}, \mathbf{2 4}$, and $\mathbf{3 3}$, that have high in vitro antiviral potencies against M2 WT and M2 L26F as well as 29, 61 and 62. EP testing confirmed that the compounds 21, 24, 61 and likely 33 are acting by blocking M2 WT channel; compound 33 seems to 
be a slow blocker as we showed previously for compound $23 .{ }^{39}$ Compounds $\mathbf{2 1}, \mathbf{2 4}, \mathbf{2 9}$, and 61 blocked efficiently M2 L26F channel. While 21, 24 and 61 blocked both M2 WT and M2 L26F channels, compound 29 is selective only for M2 L26F. Noteworthy, compound 61 is a triple inhibitor of the M2 WT, L26F and V27A mutant channels.

The observation that results showed that several of the compounds in Figure 1, such as $\mathbf{1 8}, \mathbf{2 5}, \mathbf{2 7}, \mathbf{3 7 - 4 3}, \mathbf{4 9 - 5 5}, \mathbf{5 8}, 58$ were endowed with antiviral potency against the Amt (1) or $\operatorname{Rim}(\mathbf{2})$ resistant M2 S31N A/H1N1/Calif/07/2009 virus ${ }^{28}$ can be attributed to an alternative antiviral mechanism. Their activity may be due to increasing the $\mathrm{pH}$ in endosomes. This lysomotropic effect of amantadine variants has been suggested as the mechanism ${ }^{44}$ of severe acute respiratory syndrome-coronavirus-2 (SARS-Cov-2) by amantadine decrease the viral load in positive patients suggesting that it can be prescribed as a prophylactic that prevents symptomatology caused by SARS-CoV-2 coronavirus. ${ }^{45}$

Table 4. Inhibitory effect of selected compounds on M2 WT, L26F, V27A, S31N proton channel function. ${ }^{a}$

\begin{tabular}{ccccccc}
\hline & $\mathrm{M} 2 \mathrm{WT}$ & $\mathrm{M} 2 \mathrm{~L} 26 \mathrm{~F}$ & $\mathrm{M} 2 \mathrm{~V} 27 \mathrm{~A}$ & $\mathrm{M} 2 \mathrm{~A} 30 \mathrm{~T}$ & $\mathrm{M} 2 \mathrm{S3} 1 \mathrm{~N}$ & $\mathrm{M} 2 \mathrm{G34E}$ \\
\hline $\mathbf{2 1}$ & $80.3 \pm 0$ & $93.3 \pm 0.7$ & 0 & n.t. $^{b}$ & 0 & n.t. \\
$\mathbf{2 4}$ & $79.4 \pm 0.9$ & $91.7 \pm 0.6$ & $16.5 \pm 0$ & n.t. & 0 & n.t. \\
$\mathbf{2 9}$ & 0 & $65.5 \pm 0.5$ & $1.6 \pm 1.6$ & n.t. & 0 & n.t. \\
$\mathbf{3 3}$ & $30.8 \pm 2.1$ & $12.2 \pm 1.1$ & $6.5 \pm 0.0$ & n.t. & $6.9 \pm 0$ & n.t. \\
$\mathbf{6 1}$ & $84.2 \pm 0.5$ & $79.2 \pm 1.6$ & $91.0 \pm 1.0$ & n.t. & $2.0 \pm 1.0$ & n.t. \\
$\mathbf{6 2}$ & $4.8 \pm 2.8$ & $2.9 \pm 0.1$ & 0 & n.t. & 0 & n.t. \\
\hline
\end{tabular}

${ }^{a}$ Inhibition by $100 \mu \mathrm{M}$ of compound for $2 \mathrm{~min}(\% \pm \mathrm{SE})$. Three replicates were used for measurements; n.t., not tested.

$\mathrm{M} 2$ mutants selection by drug resistance to $\mathrm{M} 2 \mathrm{WT}$

Resistance of M2 WT to Amt (1) develops rapidly in vitro, 5,46,47 in mice, ${ }^{48}$ and in the clinical setting ${ }^{49}$ through a small set of mutations, primarily L26F, V27A, V27T, A30T, S31N, and G34E. These are residues whose side chains are near the 4-fold symmetric amantadine binding site. We examined in more detail the resistance development 
pathways of H3N2 virus with M2 protein WT to a few compounds $(\mathbf{2 1}, \mathbf{2 7}, \mathbf{3 6}, \mathbf{3 8})$. We carried out passaging experiments with plaque sequencing analysis, that is, amino-acid translation of the M-segment in the presence of the compounds 21, 27, 36, 38 (Table 5).

Table 5. Mutations developing in influenza WT M2 WT a after passaging amantadine variants $\mathbf{2 1 , 2 7 ,} 36$ or 38 .

\begin{tabular}{|c|c|c|c|c|c|}
\hline $\mathrm{H} 3 \mathrm{~N} 2^{\mathrm{a}}$ & $\underset{b}{\text { Compound }}$ & 21 & 27 & 36 & 38 \\
\hline $\mathbf{P}^{c}$ & Plaque ${ }^{f}$ & $\underset{d}{1 \mu \mathrm{g} / \mathrm{ml}}$ & $\begin{array}{c}1 \\
\mu \mathrm{g} / \mathrm{ml}\end{array}$ & $\begin{array}{c}1 \\
\mu \mathrm{g} / \mathrm{ml}\end{array}$ & $5 \mu \mathrm{g} / \mathrm{ml}$ \\
\hline \multirow[t]{3}{*}{2} & 1 & $\mathrm{~V} 27 \mathrm{~A}^{e}$ & V27A & WT & A30T \\
\hline & 2 & WT & V27A & WT & A30T \\
\hline & 3 & - & V27A & WT & A30T \\
\hline \multirow[t]{4}{*}{5} & 1 & A30T* & V27A & АЗОТ & АЗ30T \\
\hline & 2 & V27A & V27A & A30V & A30T \\
\hline & 3 & V27A & V27A & АЗОТ & АЗ30T \\
\hline & & $\begin{array}{c}10 \\
\mu \mathrm{g} / \mathrm{ml}\end{array}$ & - & $\begin{array}{c}2 \\
\mu \mathrm{g} / \mathrm{ml}\end{array}$ & $5 \mu \mathrm{g} / \mathrm{ml}$ \\
\hline \multirow[t]{3}{*}{10} & 1 & V27A & - & АЗОТ & A30T \\
\hline & 2 & V27A & - & АЗОT & A30T \\
\hline & 3 & V27A & - & A30T & A30T \\
\hline \multicolumn{6}{|c|}{$\begin{array}{l}\text { a Parent strain is } \mathrm{A} / \text { Hong Kong/1/1968 (H3N2 M2 WT); } \\
\text { compound number from Figure } 1 \text {; }{ }^{c} \text { passage number; }{ }^{d} \mathrm{MDCK} \\
\text { cells were bathed in media containing the concentrations } \\
\text { specified; e sequences of resistant progeny of WT induced by } \\
\text { compounds in the top row; }{ }^{f} \text { Three separate plaques were } \\
\text { sampled and sequenced at passages } 2,5 \text {, and } 10 \text {. }\end{array}$} \\
\hline
\end{tabular}

The development of viral resistance falls into three clusters. The first cluster include compound 38, to which the WT virus rapidly develops resistance through mutation at $A 30$, particularly to threonine, suggesting that this drug block the M2 WT, but do not block M2 A30T. The second cluster include compounds that do not block V27A, ie. 21, 27, 36. These resistant strains suggest that different strains have different drug sensitivities. It may, therefore, be possible to design a cocktail of compounds to prevent resistance development for influenza A M2-blockers. These compounds cause the mutation A30T since A30 is in contact with the adduct or the mutation V27A since adamantyl cage is in contact with V27. ${ }^{8,9}$ Some of these derivatives or cocktails may be valuable based on the possibility of the virus to mutate from S31N back to S31. 
Antiviral activity against other amantadine sensitive-M2 WT and amantadine resistant-M2 S31N viruses

To investigate further the observation that amantadine variants, such as $\mathbf{3 8}, \mathbf{4 9}$, have antiviral potency against $\mathrm{A} / \mathrm{H} 1 \mathrm{~N} 1 / \mathrm{Calif} / 07 / 2009{ }^{45}$ or $\mathrm{A} / \mathrm{H} 1 \mathrm{~N} 1 /$ PuertoRico/8/1934 ${ }^{38,50}$ without blocking the M2 S31N channel, we tested compounds against the M2 S31N viruses A/H1N1/Calif/07/2009, A/H1N1/PuertoRico/8/1934, A/H1N1/WS/33 (Table 6). Also few compounds were tested against the M2 WT viruses A/H3N2/Victoria/3/75 and A2/H2N2/Taiwan/1/64. Rim (2) is inactive against A/H1N1/Calif/07/2009, A/H1N1/PR/8/1934, A/H1N1/WS/33 having M2 channel with asparagine31 but active against $\mathrm{A} / \mathrm{H} 3 \mathrm{~N} 2 /$ Victoria/3/75 and A2/H2N2/Taiwan/1/64 having M2 channel with serine-31 but also against $A / H 1 N 1 / P R / 8 / 1934$ with M2 A30T S31N. ${ }^{28,38}$

We found several low micromolar inhibitors $(18, \mathbf{2 5}, \mathbf{2 7}, \mathbf{3 7 - 4 3}, \mathbf{4 9 - 5 5}, \mathbf{5 8}, \mathbf{5 8})$ against A/H1N1/Calif/07/2009 with 27 having a submicromolar activity. Also we indentified several low micromolar against A/H1N1/PuertoRico/8/1934, which is M2 A30T/S31N virus, (2, 15, 28, 35-40, 42. $43,51,53,59$ ) with $15,28,38,39,40,42,51,53,59$ having a submicromolar activity. We observed also micromolar activities for compounds $\mathbf{3 6}, \mathbf{3 7}, \mathbf{4 0}$ against A/H1N1/WS/33. Compounds $2,17,18,28,35-40,42,43,51,53,54,56,57$ have low micromolar activity against A/H3N2/Victoria/3/75 with M2 WT channel while all compounds tested, ie. 2, 28, 35-40, 42, 43, 51, 59 have low micromolar potency against M2 WT A2/H2N2/Taiwan/1/64. Thus, several compounds blocked replication of M2 S31N viruses without blocking M2 S31N-mediated proton current for the 2-min drug treating of the M2 channel (Table 4). 
Table 6. Antiviral activity and cell cytotoxicity against influenza A M2 WT and S31N viruses.

\begin{tabular}{|c|c|c|c|c|c|}
\hline \multirow[t]{3}{*}{ Compound } & \multicolumn{5}{|c|}{$I C_{50}(\mu M)^{a}$} \\
\hline & $\begin{array}{c}\text { A/Calif/07/2009 } \\
\text { (H1N1) }\end{array}$ & $\begin{array}{c}\text { A/Victoria/3/75 } \\
\text { (H3N2) }\end{array}$ & $\begin{array}{c}\text { A2/Taiwan/1/64 } \\
\text { (H2N2) }\end{array}$ & $\begin{array}{c}\text { A/PR/8/34 } \\
\text { (H1N1) }\end{array}$ & $\begin{array}{l}\text { A/WS/33 } \\
\text { (H1N1) }\end{array}$ \\
\hline & M2 S31N & M2 WT & M2 WT & M2 A30T/S31N & M2 N31 \\
\hline 2 & $106 \pm 41$ & $0.53 \pm 0.07$ & $1.64 \pm 0.29$ & $3.26 \pm 0.5$ & $314 \pm 135$ \\
\hline 9 & $70.8 \pm 10.5$ & $72.24 \pm 10.58$ & n.t. ${ }^{b}$ & n.t. & n.t. \\
\hline 10 & $363 \pm 3.76$ & $10.16 \pm 2.58$ & n.t. & n.t. & n.t. \\
\hline 11 & $61.2 \pm 6.6$ & $39 \pm 4.5$ & n.t. & n.t. & n.t. \\
\hline 12 & $41.9 \pm 11.8$ & $91 \pm 1.5$ & n.t. & n.t. & n.t. \\
\hline 14 & $241 \pm 121$ & $105.4 \pm 35$ & n.t. & n.t. & n.t. \\
\hline 15 & $43.2 \pm 1.11$ & $33.9 \pm 7.32$ & n.t. & $0.79 \pm 0.80$ & $37.4 \pm 11.8$ \\
\hline 16 & $293 \pm 273$ & $21.71 \pm 2.19$ & n.t. & n.t. & n.t. \\
\hline 17 & $340 \pm 231$ & $11.89 \pm 2.31$ & n.t. & n.t. & n.t. \\
\hline 18 & $11.7 \pm 1$ & $3.76 \pm 0.37$ & n.t. & n.t. & n.t. \\
\hline 19 & $398 \pm 176$ & $43.6 \pm 6.07$ & n.t. & n.t. & n.t. \\
\hline 25 & $15.4 \pm 2.4$ & n.t. & n.t. & n.t. & n.t. \\
\hline 26 & $7.03 \pm 1.20$ & n.t. & n.t. & n.t. & n.t. \\
\hline 27 & $0.79 \pm 0.14$ & n.t. & n.t. & n.t. & n.t. \\
\hline 28 & $3.62 \pm 0.49$ & $2.31 \pm 0.37$ & $4.22 \pm 0.98$ & $0.3 \pm 0.5$ & $53.7 \pm 11.5$ \\
\hline 35 & $151 \pm 33^{28}$ & $3.32 \pm 0.89$ & $0.81 \pm 0.27$ & $3.76 \pm 1.02$ & $112 \pm 14.8$ \\
\hline 36 & $53.9 \pm 12.6^{28}$ & $2.02 \pm 0.43$ & $0.54 \pm 0.54$ & $0.44 \pm 0.44$ & $18.7 \pm 4.49$ \\
\hline 37 & $19.0 \pm 3^{28}$ & $2.02 \pm 0.43$ & $0.81 \pm 0.27$ & $1.81 \pm 0.94$ & $22.5 \pm 2.89$ \\
\hline 38 & $4.71 \pm 0.9228$ & $23.4 \pm 7.6$ & $0.54 \pm 0.54$ & $0.5 \pm 0.2$ & $37.6 \pm 8.4$ \\
\hline 39 & $7.42 \pm 0.91^{28}$ & $4.02 \pm 1.19$ & $1.46 \pm 0.25$ & $0.3 \pm 0.5$ & $34.6 \pm 4.1$ \\
\hline 40 & $5.75 \pm 0.37^{28}$ & $13.26 \pm 2.02$ & $0.36 \pm 0.12$ & $0.3 \pm 0.5$ & $20.5 \pm 3.7$ \\
\hline 42 & $17.4 \pm 1.41^{28}$ & $5.18 \pm 1.05$ & $0.24 \pm 0.24$ & $0.3 \pm 0.5$ & $86.0 \pm 19.6$ \\
\hline 43 & $9.72 \pm 1.38^{28}$ & $17 \pm 3.1$ & $0.24 \pm 0.32$ & $1.2 \pm 1.1$ & $26.9 \pm 14.5$ \\
\hline 46 & $399 \pm 297$ & n.t. & n.t. & n.t. & n.t. \\
\hline 47 & $30.8 \pm 49$ & n.t. & n.t. & n.t. & n.t. \\
\hline 49 & $8.1 \pm 2.1$ & n.t. & n.t. & n.t. & n.t. \\
\hline 50 & $7.92 \pm 1.51$ & n.t. & n.t. & n.t. & n.t. \\
\hline 51 & $2.66 \pm 0.33$ & $0.56 \pm 0.21$ & $0.48 \pm 0.24$ & $0.3 \pm 0.5$ & $34.2 \pm 8.4$ \\
\hline 52 & $7.2 \pm 2$ & $55.3 \pm 0.5$ & n.t. & n.t. & $9.60 \pm 1.40$ \\
\hline 53 & $10.2 \pm 1.2$ & $5.67 \pm 1.80$ & n.t. & $0.79 \pm 0.80$ & - \\
\hline 54 & $9.5 \pm 1.6$ & $19.2 \pm 3.5$ & n.t. & n.t. & n.t. \\
\hline 55 & $7.0 \pm 0.8$ & $58.3 \pm 11.5$ & n.t. & n.t. & n.t. \\
\hline 56 & $34.2 \pm 4.4$ & $3.2 \pm 2$ & n.t. & n.t. & n.t. \\
\hline 57 & $7.7 \pm 2$ & $16.2 \pm 7.5$ & n.t. & n.t. & n.t. \\
\hline 58 & $8.7 \pm 1.7$ & $38.5 \pm 1$ & n.t. & n.t. & n.t. \\
\hline 59 & $34.1 \pm 1.2$ & $34.1 \pm 7.9$ & $1.21 \pm 0.25$ & $0.7 \pm 0.5$ & $14.9 \pm 1.6$ \\
\hline 60 & $19.8 \pm 17.1$ & n.t. & n.t. & n.t. & n.t. \\
\hline
\end{tabular}

${ }^{a} \mathrm{EC}_{50} \pm$ its standard error from mini-plaque testing for dose-response or single-dose screens, using cultured MDCK cells, based on least-squares fitting of single-site binding curves. No microscopic evidence of cytotoxicity to MDCK cells was detected after 18 hour exposure at $50 \mu \mathrm{M}$ except with compound 41, where a $5 \mu \mathrm{M}$ dose was used instead; ${ }^{b}$ n.t.; not tested. 
Development of resistance to amantadine-sensitive A/Victoria/3/75 (H3N2, M2 WT) and amantadine-resistant A/Calif/07/2009 (H1N1, M2 S31N) to amantadine variants

Resistance testing with semi-weekly passages in MDCK cell cultures was performed for amantadine 1 against an amantadine-sensitive H3N2 virus; and for compound $\mathbf{3 8}$ and a cocktail of 26, 27, and 60 against amantadine-resistant H1N1 (2009) (Table 7).

The cocktail components were selected to represent a diverse, random set of ring adducts at both $\mathrm{C} 1$ and $\mathrm{C} 2$. In the Amt (1) - H3N2 system, drug resistance appeared after one passage in the presence of drug, with no detectable activity of Amt (1) against the progeny from passage 1 or passage 2 at $50 \mu \mathrm{M}$, but normal Amt (1) activity against the original virus post hoc $\left(\mathrm{EC}_{50} 3.0 \pm 0.5\right.$ $\mu \mathrm{M} \mathrm{N}=9$ ). In contrast, in the $38-\mathrm{H} 1 \mathrm{~N} 1$ system, virus progeny produced in the presence of drug at passages 1-5 maintained full drug sensitivity ( $\mathrm{EC}_{50}$ 2.1-5.4 $\left.\mu \mathrm{M}\right)$. Resistance to $\mathbf{3 8}$ developed between passage 6 and passage 12, becoming significant after passage 10. Likewise, the cocktail of 26, 27, and 60 remained effective through 6 passages, but the H1N1 virus developed resistance by passage 10.

Table 7. Resistance testing for amantadine 1 against an amantadine-sensitive H3N2 virus and potent amantadine variants against amantadine-resistant H1N1 (2009) (see Table 6).

\begin{tabular}{|c|c|c|c|}
\hline Passage \# & $\begin{array}{c}1(5 \mu \mathrm{M}) \\
\text { A/Victoria/3/75 } \\
\text { (H3N2, M2 WT) } \\
\text { EC }_{50 \pm \text { S.E. }(\mu \mathrm{M})}\end{array}$ & $\begin{array}{c}38(5 \mu \mathrm{M}) \\
\text { A/Calif/07/2009 } \\
(\mathrm{H} 1 \mathrm{~N} 1, \mathrm{M} 2 \mathrm{~S} 31 \mathrm{~N}) \\
\mathrm{EC}_{50} \pm \text { S.E. }(\mu \mathrm{M}) \\
\end{array}$ & $\begin{array}{c}\mathbf{2 6 , 2 7 , 6 0}(5 \mu \mathrm{M}) \\
\text { A/Calif/07/2009 } \\
(\mathrm{H} 1 \mathrm{~N} 1, \mathrm{M} 2 \mathrm{~S} 31 \mathrm{~N}) \\
\mathrm{EC}_{50} \pm \text { S.E. }(\mu \mathrm{M})\end{array}$ \\
\hline 0 & $2.77 \pm 0.29$ & $4.71 \pm 0.92$ & $0.99 x \pm 0.15$ \\
\hline 1 & Inactive & $5.4 \pm 1.4$ & - \\
\hline 2 & Inactive & $3.7 \pm 0.5$ & - \\
\hline 5 & N.D. & $2.1 \pm 1.6$ & - \\
\hline 6 & N.D. & - & $1.20 x \pm 0.07$ \\
\hline 8 & N.D. & $18.5 \pm 1.0$ & - \\
\hline 10 & N.D. & $76 \pm 9$ & $7.9 x \pm 0.8$ \\
\hline 12 & N.D. & $149 \pm 115$ & n.t. \\
\hline Drug 29 & & $10.2 \pm 1.7$ & \\
\hline
\end{tabular}

n.t.; not tested. 
The passage-12 38-resistant mutant was subsequently tested and found to be sensitive to 28 (ie. the $\mathrm{EC}_{50}$ modestly reduced at $10.2 \mu \mathrm{M}$ compared to $3.6 \mu \mathrm{M}$ against $\mathrm{H} 1 \mathrm{~N} 1$ (2009)). Without any drug in the medium, the development of viral resistance to compound $\mathbf{3 8}$ was negligible, ie. the $\mathrm{EC}_{50}$ retested at passage 0 was $4.7 \pm 0.7 \mu \mathrm{M}$, at passage 10: $3.0 \pm 0.3$, and at passage $30: 7.7 \pm 0.6$ $\mu \mathrm{M}$.

The compound that was very successful against H1N1 (2009), 38, induced resistance in the WT within 2 passages. As was published before, no changes from the parent A/California/07/2009 were observed for the amino-acid translation of the M-segment of the passage-12 38-resistant strain for residues sequenced, 10-73. Hence, resistance did not develop by selection of additional amantadine-resistance mutations in $\mathrm{M} 2$. The lack of sequence changes for $\mathrm{M} 2(\mathrm{~S} 31 \mathrm{~N})$-bearing virus in the presence of compound $\mathbf{3 8}$ mentioned above indicates that $\mathrm{M} 2(\mathrm{~S} 31 \mathrm{~N})$-bearing virus has a different escape route than M2(WT)-bearing virus. In the latter case changes inside the M2 pore confer resistance while in the former no mutations were observed in the $\mathrm{M} 2$ channel amantadine binding-site and therefore some other change in the virus is implicated.

Mechanism of action of amantadine variants against amantadine-resistant S31N viruses investigated

Pre-exposure of virus to drug before cells infection

We also performed experiments with A/Calif/07/09 inhibition to show that this virus inhibition does not take place at late stages of virus endocytosis M2 protein function compared to other viruses. We showed that $\mathbf{3 8}$ is capable of blocking virus infection when drug exposure occurs prior to virus access to cells, presumably virus blocking occurs on the viral envelope-associated $\mathrm{M} 2$ protein. 
EP experiments for 30-minute exposure of M2 S31N with (and without) compound 38

To provide additional evidence that amantadine variants did not block M2 S31N at latter stage of infection we performed a 30-minute exposure EP experiment with and without compound 38 (Figure 2). The results revealed no blocking of $\mathrm{M} 2 \mathrm{~S} 31 \mathrm{~N}$ by 38 during the $30 \mathrm{~min}$ exposure.

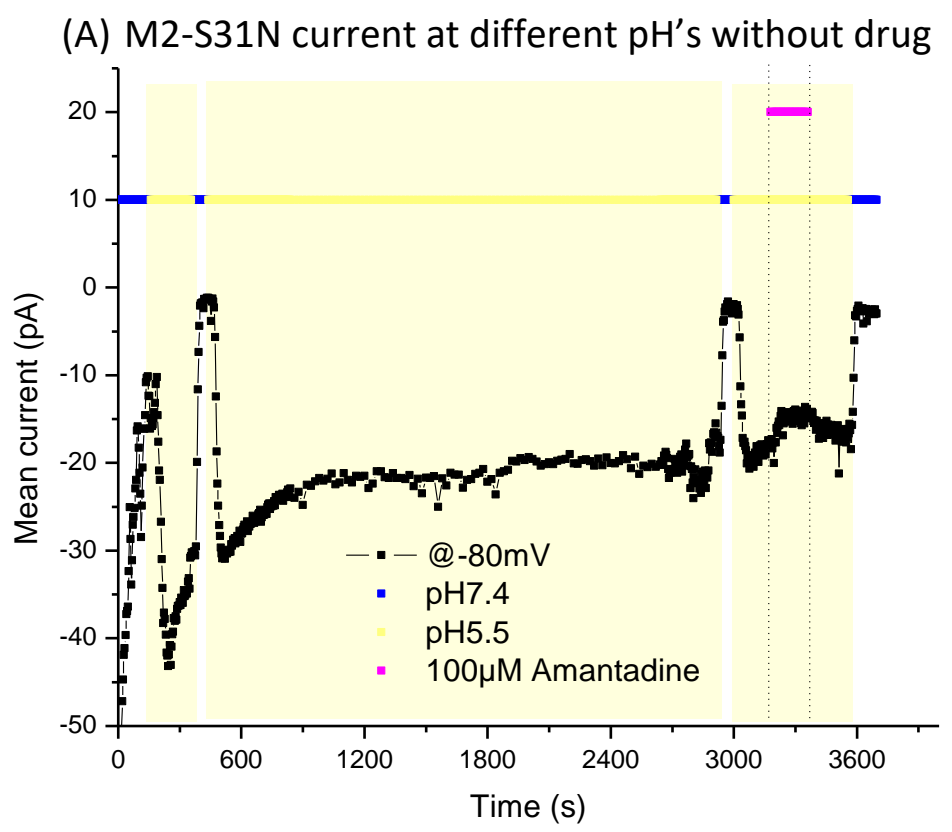

(B) M2-S31N current at different $\mathrm{pH}$ 's with compound 38

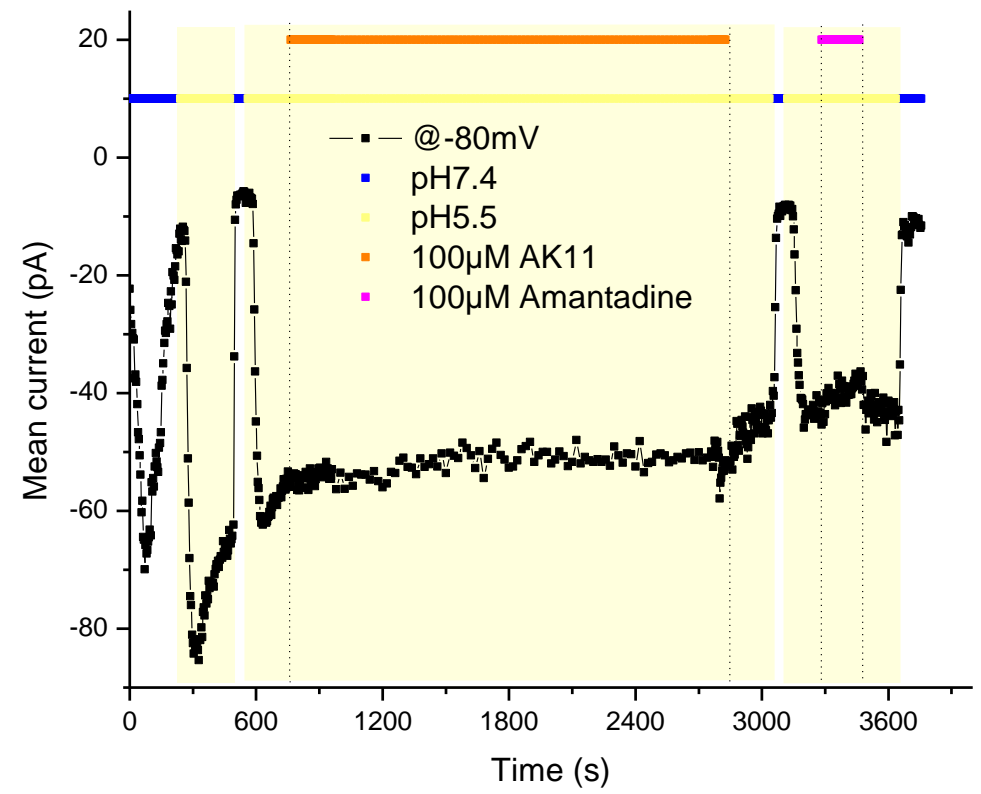

Figure 2. Panels (A), (B) show the 30-minute exposure of $M 2$ S31N without or with 38, respectively. 
Since the sequences of WSN/33/H1N1 and A/California/07/2009/H1N1 differ just on residue 43 of M2 (43T and 43L respectively, see Table S1) ${ }^{50}$ another target seems to interfere with amantadine variants blocking A/California/07/2009/H1N1 and other S31N viruses (see Tables 6 S2).

Binding of haemagglutinin to the virus

In recent papers, we suggested that haemagglutinin (HA) can be involved for the antiviral activity of amantadine variants to viruses (including some M2 S31N viruses) and find mutations of HA in aminoacids that may influence either HA binding to cell receptors or possibly the efficiency of the conformational changes that may take place at lower $\mathrm{pH} .{ }^{28,37,38,51}$

We performed additional experiments to attempt identifying the stage at which virus propagation is blocked. HA-inhibition assays were used to investigate whether amantadine variants inhibited receptor binding (see Supporting Information). A haemolysis assay was used to see whether the compounds inhibited the acid induced fusion activity of the virus in a similar manner to the antivirals Arbidol (Umifenovir) ${ }^{52}$ or tertiary butylhydroquinone (TBHQ). ${ }^{53}$ These drugs inhibit between the viral envelope and the cell membrane of the target cell by preventing contact between the virus and target host cells which prevents viral entry to the target cell, and therefore protects it from infection. With APR8 virus and compound 38, which had been shown to be very efficient at inhibiting propagation of this virus (Table 6), there was no detectable inhibition of haemolytic activity compared with control. Therefore, the block in viral propagation seems that it does not occur at the virus-endosomal membrane fusion stage.

Endosome neutralizers

To explore further the possibility of a mechanism of antiviral activity of amantadine variants against some $M 2 S 31 N$ viruses $28,37,38,51$ with implication of $H A$, we thought that amantadine variants may increase the endosomal $\mathrm{pH}$ and thus indirectly inhibit the $\mathrm{HA}$ conformational change which is required for fusion. We selected virus mutants resistant to the amantadine variants, and 
they had an increased fusion $\mathrm{pH}$ and mutations in $\mathrm{HA}$. Also, when we compared a few H1N1 strains (including H1N1pdm09), their sensitivity to the amantadine variants correlated with their fusion $\mathrm{pH}$. These results suggested that HA can represent a sensitivity factor. ${ }^{11,37,51}$ However, the intriguing observation that X-31 (A/Aichi/2/1968) and APR8 had the same fusion $\mathrm{pH}(5.0$; which is low compared to other strains), and yet X-31 is totally insensitive to amantadine variants, while APR8 has very robust sensitivity, seems to be contradictory. Perhaps H1 HA is more sensitive to this $\mathrm{pH}$ effect compared to H3 HA. ${ }^{11,37,51}$

In this work we also showed that Bovine papillomavirus (BPV) infection of Bovine embryonic kidney cells is not inhibited by compounds $\mathbf{3 8}$ (which is submicromolar inhibitor of A/PR/8/34 having M2 S31N/A30T) and 27 (which is submicromolar inhibitor of A/Calif/07/09 having M2 S31N) in the 1-20 $\mu \mathrm{M}$ range (see Supporting Information) even though it is inhibited by modest concentrations of endosome neutralizers (lysosomotropic efect), chlorpromazine, ammonium, chloroquine, and bafilamycin A1. This argues that $\mathbf{3 8}$ and $\mathbf{2 7}$ are not good endosome neutralizers lacking a chloroquine effect. ${ }^{54}$ Additionally it has been found that chloroquine effect alone (endosomal or perhaps viral alkalinization) works in cell culture but not in humans. ${ }^{55}$

\section{Neurotoxicity study}

Compounds tested here might be useful for re-purposing of amantadine class for compounds not only because of antigenic shift of the virus to M2 WT but also because the compounds tested here and cocktails inhibit a broad panel of viruses. There was no evidence of cytotoxicity (inhibition of cell growth or cell morphological changes) for any of the compounds at dosage $\leq 50 \mu \mathrm{M}$. Toxicity of compounds $\mathbf{3 8}$ and $\mathbf{4 9}$ were assessed in CD-1 mice and compared to amantadine $\mathbf{1}$ (Table 8).

Intraperitoneal doses of all three compounds were non-lethal at $30 \mathrm{mg} / \mathrm{kg}$. Compound 38 was lethal to all 3 mice tested at $100 \mathrm{mg} / \mathrm{kg}$ and 1 and 49 were lethal at $300 \mathrm{mg} / \mathrm{kg}$. Some signs of neural effects were seen with $\mathbf{4 9}$ at $30 \mathrm{mg} / \mathrm{kg}$, but not with 1 and $\mathbf{3 8}$. 
Table 8. Toxicity from I.P. injections of CD-1 mice.

\begin{tabular}{|l|l|l|l|}
\hline Dose $(\mathrm{mg} / \mathrm{kg})$ & Amantadine 1 & Compound 18 & Compound 31 \\
\hline 30 & $0(2: 2)^{\mathrm{a}}$ & $0(1: 2)$ & $0(2: 1)^{\mathrm{b}}$ \\
\hline 100 & $0(2: 1)^{\mathrm{c}}$ & $3(2: 1)$ & $2(3: 1)^{\mathrm{d}}$ \\
\hline 300 & $3(2: 1)$ & N.D. & $3(2: 1)$ \\
\hline
\end{tabular}

${ }^{a}$ Number of deaths within 48 hours (number of males injected: number of females injected). With compound 8 at $100 \mathrm{mg} / \mathrm{kg}$, one male and one female died. ${ }^{\mathrm{b}}$ Slightly abnormal gate. Some quivers. Hyper-responsive to touch or noise. " Moderate trembling or quivers. ${ }^{d}$ Ataxic gait. Moderately abnormal gait. Slightly or somewhat impaired mobility. Mild tremors. Motor incoordination.

\section{Discussion}

Here, we provided a determined study for 57 synthetic amantadine variants using anti-viral assay against influenza $A$ virus with $\mathrm{M} 2 \mathrm{WT}$ and all amantadine resistant M2 mutant strains. We reported activities against WSN/33-M2 N31S covering a range of over three digits (0.03 to 31.33 $\mu \mathrm{M})$. Changes in structure of adamantane, which are often subtle, result in remarkable changes in blocking efficiency for M2 channels and potency against the corresponding influenza A M2 strains. Several compounds from Kolocouris lab, eg 21, 24, block both M2 WT and M2 L26F-mediated proton current and these viruses replication, while 62 is a triple blocker of M2 WT, M2 L26F and M2 V27A channels and these viruses replication, and 29 only blocks M2 L26F channel and virus replication. We evaluated that their mechanism of inhibition is M2 blocking by EP and identified consistent point mutations for representative compounds against M2 WT viruses. Compounds 29 and 62 developed from Vazquez may provide useful probe molecules for these $M 2$ channels and corresponding viruses inhibition.

The difference in the anti-viral potency of the amantadine variants against A/WSN/33 virus and $\mathrm{A} /$ Calif/09 or $\mathrm{A} / \mathrm{PR} / 8 / 34$ viruses with asparagine at position 31 of $\mathrm{M} 2$ (and possibly M2 WT viruses) that was shown and the lack of M2 S31N blocking activity is not understood. ${ }^{11,38,28}$ Our previous results showed also that aminoadamantane-aryl group conjugates are active even against the WSN/33, ${ }^{56}$ without blocking the M2 S31N channel nor bind HA and their mechanism of action needs further investigation. These findings suggested additional mechanisms of anti-viral activity, 
plausibly a lysosomotropic effect for amantadine variants with lipophilic adducts inhibiting virus at concentrations usually higher than ca. $5 \mu \mathrm{M} .{ }^{11,38,28,56}$ These compounds are lipophilic and can be accumulated and buffer late-stage endosomes to prevent acid-induced fusion of the virus envelope with the endosome membrane. ${ }^{57}$ Such lysosomotropic agents, like chloroquine, are effective against many types of viruses in vitro, possibly including also the SARS-CoV-2. ${ }^{12}$

However, we showed that the mechanism of antiviral activity against A/Calif/09 or A/PR/8/34 and possibly also M2 WT viruses compared to WSN/33 viruses is not due to inhibition of an early stage of virus infection or a late stage of $M 2$ channel function during endocytosis or inhibition of HA binding to host cells or a different $\mathrm{pH}$ for HA fusion or a lysosomotropic effect. If these additional mechanisms will be rationalized, this knowledge may be used to develop antivirals to block other viruses including the SARS-CoV-2. It should be emphasized that we did not explore if the drugs can inhibit another stage of entry of the virus into a new host cell, where M1 protein must be dissociated from the RNPs, allowing them to enter the nucleus. This event allows the import of incoming RNPs into the nucleus. ${ }^{58}$ Thus, intraviral buffering inhibiting M1-RNP disruption could be an adamantane mechanism-of-action.

We provided a neurotoxicity study in mice for selected compounds showing that variants other than amantadine showed similar toxicity profile. The compounds and their cocktails while not to be more toxic than amantadine might be useful for re-purposing of amantadine class of drugs because: (a) they inhibited a broad panel of M2 WT (and can be useful in the case of an antigenic shift of the virus to M2 WT) and M2 S31N viruses including the H1N1pdm09, and (b) they can inhibit M2 L26F or even M2 V27A strains that in the future can be prevalent virus strains. 


\section{Conclusion}

The results for amantadine variants against influenza A presented here provide a reference study. We reported activities for 57 compounds against WSN/33-M2 N31S covering a range of over three digits $(0.03$ to $31.33 \mu \mathrm{M})$. Several compounds block in TEVC experiments both M2 WT and M2 L26F-mediated proton current and the corresponding viruses replication. We identified one triple blocker of M2 WT, M2 L26F and M2 V27A channels and viruses replication a compound that only blocks M2 L26F channel and virus replication. With WT viruses, which more readily developed resistance to these compounds, the pattern of resistant $M 2$ sequence changes suggests that mutants have specific drug sensitivities, which could lead the way to the development of impenetrable cocktails. We paid an effort to explore features that may be responsible for the fact that some amantadine variant inhibited $\mathrm{A} /$ Calif/09 or A/PR/8/34 without blocking M2 S31N channel-mediated proton current compared to WSN/33 viruses. We found that this is not due to inhibition of an early stage of virus infection or a late stage of $\mathrm{M} 2$ channel function during endocytosis or inhibition of $\mathrm{HA}$ binding to host cells or a different $\mathrm{pH}$ for $\mathrm{HA}$ fusion or a lysosomotropic effect. However, these compounds and their cocktails while not to be more toxic than amantadine might be useful in the case a re-purposing of amantadine class of drugs will be needed.

\section{Supporting Information}

Synthetic chemistry methods and experimental methods for chemical synthesis and experimental methods for biological evaluation and neurotoxicity data, sequences alignments of $\mathrm{M} 2$ proteins from different viruses and SI references (six Schemes and two Tables). 


\section{Author Information}

"Corresponding author: Antonios Kolocouris, Panepistimiopolis-Zografou, Athens 15771, Greece; Phone: (+301) 210-7274834, E-mail: ankol@pharm.uoa.gr

AK designed this research project which includes the graduate project thesis of MS. MS, IS, CT, CL in AK group synthesized compounds; MS and CT contributed equally. ALT in SV group resynthesized compounds 29, 61, 62. EP experiments were performed by CM in JW lab (see Table 4) and by DF group (see Figure 2). Antiviral testing against WSN viruses was performed by AH, PS, KD in MS group and against other viruses by BJ. RZ performed the point mutations experiments. IA, FS performed the neurotoxicity experiments. SW did the HA binding experiments. AK wrote the manuscript and MS, ALT, SV revised it.

\section{Acknowledgements}

We thank Chiesi Hellas for supporting this research, Professor Nikolas Kolokouris for providing samples of compounds 46 and 47, Assistant Professor loannis Papanastasiou for providing a sample of compound 32, $\mathrm{Dr}$ Antonios Drakopoulos for its help on synthesis of compound 30, $\mathrm{Dr}$ Natalie A. Fokina and Professor Peter R. Schreiner_for providing a sample of compound $\mathbf{3 4}$. Andreea L. Turcu thanks the Spanish Ministerio de Educacion, Cultura y Deporte for a PhD fellowship (FPU program). Antonios Kolocouris and Santiago Vázquez thank Fundació La Marató de TV3 (201832) for financial support. The authors thank Dr. Donald Smee for providing Influenza A/California/04/2009 (H1N1). 


\section{References}

1. Wang, C., Takeuchi, K., Pinto, L. H. \& Lamb, R. A. Ion channel activity of influenza A virus M2 protein: characterization of the amantadine block. J. Virol. 67, 5585-94 (1993).

2. Chizhmakov, I. V et al. Selective proton permeability and $\mathrm{pH}$ regulation of the influenza virus M2 channel expressed in mouse erythroleukaemia cells. J. Physiol. 494, 329-336 (1996).

3. Hayden, F. G. Clinical applications of antiviral agents for chemophrophylaxis and therapy of respiratory viral infections. Antiviral Res. Suppl 1, 229-39 (1985).

4. Griffin, S. D. C. et al. The p7 protein of hepatitis C virus forms an ion channel that is blocked by the antiviral drug, Amantadine. in FEBS Letters 175-184 (2003). doi:10.1016/S00145793(02)03851-6

5. Cady, S. D. et al. Structure of the amantadine binding site of influenza M2 proton channels in lipid bilayers. Nature 463, 689-692 (2010).

6. Wright, A. K. et al. Differential Binding of Rimantadine Enantiomers to Influenza A M2 Proton Channel. J. Am. Chem. Soc. 138, 1506-1509 (2016).

7. Ma, C. et al. Identification of the functional core of the influenza A virus A/M2 protonselective ion channel. Proc Natl Acad Sci U S A 106, 12283-12288 (2009).

8. Thomaston, J. L. et al. Inhibitors of the $\mathrm{M} 2$ proton channel engage and disrupt transmembrane networks of hydrogen-bonded waters. J. Am. Chem. Soc. 15219-15226 (2018). doi:10.1021/jacs.8b06741

9. Thomaston, J. L. et al. X-ray Crystal Structures of the Influenza M2 Proton Channel DrugResistant V27A Mutant Bound to a Spiro-Adamantyl Amine Inhibitor Reveal the Mechanism of Adamantane Resistance. Biochemistry 59, 627-634 (2020).

10. Pisonero-Vaquero, S. \& Medina, D. L. Lysosomotropic Drugs: Pharmacological Tools to Study Lysosomal Function. Curr. Drug Metab. 18, 1147-1158 (2017).

11. Scholtissek, C., Quack, G., Klenk, H. D. \& Webster, R. G. How to overcome resistance of influenza A viruses against adamantane derivatives. Antiviral Res. 37, 83-95 (1998).

12. Wang, M. et al. Remdesivir and chloroquine effectively inhibit the recently emerged novel coronavirus (2019-nCoV) in vitro. Cell Res. 30, 269-271 (2020). 
13. Skehel, J. J., Hay, A. J. \& Armstrong, J. A. On the Mechanism of Inhibition of Influenza Virus Replication by Amantadine Hydrochloride. J. Gen. Virol. 38, 97-110 (1978).

14. Hay, A. J., Wolstenholme, A. J., Skehel, J. J. \& Smith, M. H. The molecular basis of the specific anti-influenza action of amantadine. EMBO J. 4, 3021-3024 (1985).

15. Krumbholz, A. et al. High prevalence of amantadine resistance among circulating European porcine in fluenza A viruses. J. Gen. Virol. 90, 900-908 (2009).

16. Stouffer, A. L. et al. The Interplay of Functional Tuning, Drug Resistance, and Thermodynamic Stability in the Evolution of the M2 Proton Channel from the Influenza A Virus. Structure (2008). doi:10.1016/j.str.2008.04.011

17. Astrahan, P. \& Arkin, I. T. Resistance characteristics of influenza to amino-adamantyls. Biochim. Biophys. Acta - Biomembr. 1808, 547-553 (2011).

18. Furuse, Y., Suzuki, A., Kamigaki, T. \& Oshitani, H. Evolution of the M gene of the influenza A virus in different host species: large-scale sequence analysis. Virol. J. 6, 67 (2009).

19. Bright, R. A. et al. Incidence of adamantane resistance among influenza A (H3N2) viruses isolated worldwide from 1994 to 2005: A cause for concern. Lancet 366, 1175-1181 (2005).

20. Lan, Y. et al. A comprehensive surveillance of adamantane resistance among human influenza A virus isolated from mainland China between 1956 and 2009. Antivir. Ther. 15, 853-859 (2010).

21. Dong, G. et al. Adamantane-resistant influenza a viruses in the world (1902-2013): Frequency and distribution of M2 gene mutations. PLoS One 10, 1-20 (2015).

22. Garcia, V. \& Aris-Brosou, S. Comparative dynamics and distribution of influenza drug resistance acquisition to protein $\mathrm{M} 2$ and neuraminidase inhibitors. Mol. Biol. Evol. 31, 355363 (2014).

23. Furuse, Y., Suzuki, A. \& Oshitani, H. Large-scale sequence analysis of M gene of influenza A viruses from different species: Mechanisms for emergence and spread of amantadine resistance. Antimicrob. Agents Chemother. 53, 4457-4463 (2009).

24. Aldrich, P. E. et al. Antiviral Agents. 2. Structure-Activity Relationships of Compounds Related to 1-Adamantanamine. J. Med. Chem. 14, 535-543 (1971).

25. Kolocouris, N. et al. Synthesis and antiviral activity evaluation of some aminoadamantane derivatives. J Med Chem 37, 2896-2902 (1994). 
26. Stamatiou, G. et al. Heterocyclic rimantadine analogues with antiviral activity. Bioorganic Med. Chem. 11, (2003).

27. Zoidis, G. et al. Are the 2-isomers of the drug rimantadine active anti-influenza A agents? Antivir. Chem. Chemother. 14, (2003).

28. Kolocouris, A. et al. Aminoadamantanes with persistent in vitro efficacy against H1N1 (2009) influenza A. J. Med. Chem. 57, 4629-4639 (2014).

29. Balannik, V. et al. Functional studies and modeling of pore-lining residue mutants of the influenza A virus M2 ion channel. Biochemistry 49, 696-708 (2010).

30. Wang, J. et al. Exploring the Requirements for the Hydrophobic Scaffold and Polar Amine in inhibitors of M2 from Influenza A Virus. ACS Med. Chem. Lett. 2, 307-312 (2011).

31. Rey-Carrizo, M. et al. Easily accessible polycyclic amines that inhibit the wild-type and amantadine-resistant mutants of the M2 channel of influenza A virus. J. Med. Chem. 57, 5738-5747 (2014).

32. Rey-Carrizo, M. et al. New polycyclic dual inhibitors of the wild type and the V27A mutant M2 channel of the influenza A virus with unexpected binding mode. Eur. J. Med. Chem. 96, 318-329 (2015).

33. Wang, J. et al. Molecular Dynamics Simulation Directed Rational Design of Inhibitors Targeting Drug-Resistant Mutants of Influenza A Virus M2. J. Am. Chem. Soc. 133, 12834$12841(2011)$

34. Wang, J. J. et al. Structure and inhibition of the drug-resistant S31N mutant of the M2 ion channel of influenza A virus. Proc Natl Acad Sci U S A 110, 1315-1320 (2013).

35. Wang, Y. et al. In Vitro Pharmacokinetic Optimizations of AM2-S31N Channel Blockers Led to the Discovery of Slow-Binding Inhibitors with Potent Antiviral Activity against DrugResistant Influenza A Viruses. J. Med. Chem. 61, 1074-1085 (2018).

36. Kolocouris, N. et al. Synthesis and antiviral activity evaluation of some new aminoadamantane. J Med Chem 39, 3307-3318 (1996).

37. Torres, E. et al. Role of the viral hemagglutinin in the anti-influenza virus activity of newly synthesized polycyclic amine compounds. Antiviral Res. 99, 281-291 (2013).

38. Torres, E. et al. Synthesis and anti-influenza a virus activity of 2,2-dialkylamantadines and related compounds. ACS Med. Chem. Lett. 3, 1065-1069 (2012). 
39. Drakopoulos, A. et al. Unraveling the Binding, Proton Blockage, and Inhibition of Influenza M2 WT and S31N by Rimantadine Variants. ACS Med. Chem. Lett. 9, 198-203 (2018).

40. Tzitzoglaki, C. et al. Binding and Proton Blockage by Amantadine Variants of the Influenza M2WT and M2S31N Explained. J. Med. Chem. 60, 1716-1733 (2017).

41. Schade, D. et al. Zanamivir Amidoxime- and N-Hydroxyguanidine-Based Prodrug Approaches to Tackle Poor Oral Bioavailability. J. Pharm. Sci. 104, 3208-3219 (2015).

42. Drakopoulos, A. et al. Affinity of Rimantadine Enantiomers against Influenza A/M2 Protein Revisited. ACS Med. Chem. Lett. 8, 145-150 (2017).

43. Tzitzoglaki, C. et al. Binding and Proton Blockage by Amantadine Variants of the Influenza M2WT and M2S31N Explained. J. Med. Chem. 60, 1716-1733 (2017).

44. Smieszek, S. P., Przychodzen, B. P. \& Polymeropoulos, M. H. Amantadine disrupts lysosomal gene expression: A hypothesis for COVID19 treatment. Int. J. Antimicrob. Agents 55, 106004 (2020).

45. Aranda-Abreu, G. E., Aranda-Martínez, J. D. \& Araújo, R. Use of amantadine in a patient with SARS-CoV-2. Journal of Medical Virology (2021). doi:10.1002/jmv.26179

46. Cochran, K. W., Maassab, H. F., Tsunoda, A. \& Berlin, B. S. Studies on the antiviral activity of amantadine hydrochloride. Ann. N. Y. Acad. Sci. 130, 432-439 (1965).

47. Appleyard, G. Amantadine resistance as a genetic marker for influenza viruses. J. Gen. Virol. 36, 249-255 (1977).

48. Oxford, J. S., Logan, I. S. \& Potter, C. W. Studies on the antiviral activity of amantadine hydrochloride. Ann. N. Y. Acad. Sci. 173, 300-313 (1970).

49. Hayden, F. G. et al. Recovery of drug-resistant influenza A virus during therapeutic use of rimantadine. Antimicrob. Agents Chemother. 35, 1741-1747 (1991).

50. Roberts, K. L., Leser, G. P., Ma, C. \& Lamb, R. A. The Amphipathic Helix of Influenza A Virus M2 Protein Is Required for Filamentous Bud Formation and Scission of Filamentous and Spherical Particles. J. Virol. 87, 9973-9982 (2013).

51. Vanderlinden, E. \& Naesens, L. Emerging Antiviral Strategies to Interfere with Influenza Virus Entry. Med. Res. Rev. (2014). doi:10.1002/med.21289

52. Leneva, I. A., Russell, R. J., Boriskin, Y. S. \& Hay, A. J. Characteristics of arbidol-resistant mutants of influenza virus: Implications for the mechanism of anti-influenza action of 
arbidol. Antiviral Res. (2009). doi:10.1016/j.antiviral.2008.10.009

53. Bodian, D. L. et al. Inhibition of the fusion-inducing conformational change of influenza hemagglutinin by benzoquinones and hydroquinones. Biochemistry 32, 2967-2978 (1993).

54. Shibata, M. et al. Mechanism of uncoating of influenza B virus in MDCK cells: Action of chloroquine. J. Gen. Virol. (1983). doi:10.1099/0022-1317-64-5-1149

55. Paton, N. I. et al. Chloroquine for influenza prevention: A randomised, double-blind, placebo controlled trial. Lancet Infect. Dis. 11, 677-683 (2011).

56. Tzitzoglaki, C. et al. Chemical Probes for Blocking of Influenza A M2 Wild-type and S31N Channels. ACS Chem. Biol. 15, 2331-2337 (2020).

57. Helenius, A. Unpacking the incoming influenza virus. Cell 69, 577-578 (1992).

58. Martin, K. \& Helenius, A. Nuclear transport of influenza virus ribonucleoproteins: The viral matrix protein (M1) promotes export and inhibits import. Cell 67, 117-130 (1991). 


\section{TOC Graphic}

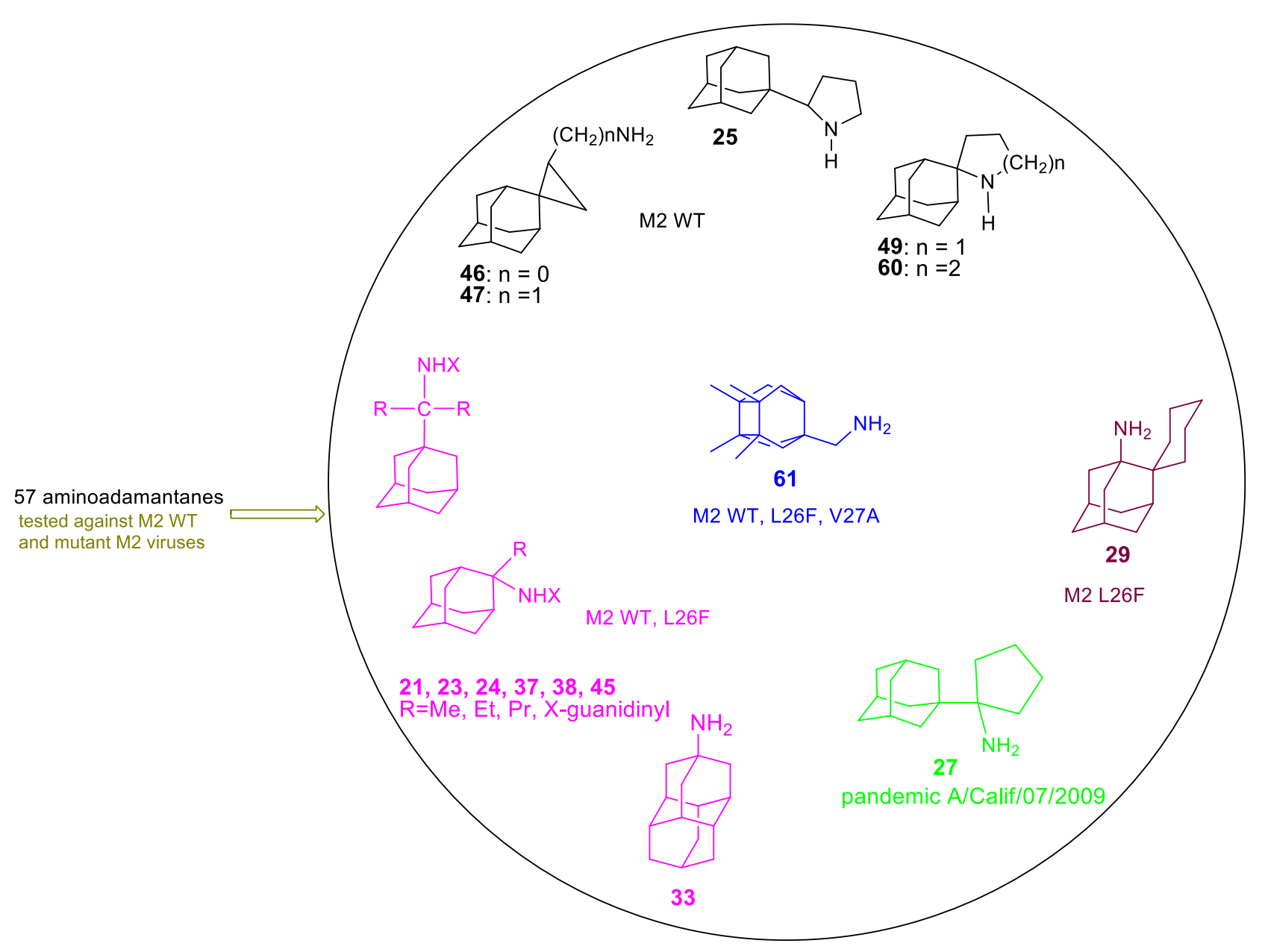

